\title{
Fortalecimiento de la competencia matemática resolución de problemas en educación básica secundaria, mediante el aprendizaje basado en problemas (ABP)
}

\section{Strengthening of the mathematics competence problem solving in high school education, through learning based on problems (LBP)}

\author{
Fortalecimento da resolução de problemas de competência matemática no ensino fundamental \\ secundário, através da aprendizagem baseada em problemas (PBL)
}

\author{
Sergio Andrés Páez - Rolón ${ }^{\mathrm{a}^{*}}$ \\ Magister en Práctica pedagógica, Docente Ministerio de Educación Nacional. Cúcuta, Colombia. \\ Orcid: 0000-0002-7992-9976
}

\begin{abstract}
Forma de citar: Páez, S. (2017). Fortalecimiento de la competencia matemática resolución de problemas en educación básica secundaria, mediante el aprendizaje basado en problemas (ABP). Eco matemático 8(1). 25-33
\end{abstract}

Recibido: marzo 22 de 2016

Aceptado: julio 03 de 2016

\section{Palabras clave \\ Competencia Matemática, Metodología Aprendizaje Basado en Problemas, Resolución de problemas}

\section{Keywords}

Mathematics Competence, Methodology, Learning Based on Problems, Problem Solving.

\begin{abstract}
Resumen: En la enseñanza de las matemáticas es indispensable la implementación de estrategias que permitan ligar al estudiante con su contexto inmediato, a fin de que pueda desarrollar en forma significativa su aprendizaje. La presente investigación se desarrolla con base en la metodología Aprendizaje Basado en Problemas (ABP), con el objetivo de analizar de qué manera influye en el fortalecimiento de la competencia matemática resolución de problemas. Llevado a cabo bajo un enfoque cuantitativo y un diseño pre-experimental, se realizó un análisis descriptivo de la habilidad de los estudiantes para resolver problemas previos a la metodología, luego, se compararon los resultados tanto individual como grupal obtenidos en el test y el post test; y con base en estos, se determinó en qué aspectos dicha metodología fortalece la competencia de resolución de problemas en estudiantes de educación básica secundaria. Los resultados permitieron concluir que se pudo fortalecer la competencia de resolución de problemas en el área de matemáticas, en el grado noveno, viéndose reflejado en el mejoramiento de las habilidades de los estudiantes para resolver problemas sobre área y volúmenes en figuras geométricas fundamentales. Lo anterior evidenciado en los resultados del post test, esto sirve como base para que los estudiantes le den sentido a los nuevos conocimientos, promoviendo la indagación en contextos reales, facilitando el aprendizaje significativo y generando cambios en el nivel de razonamiento.
\end{abstract}

\footnotetext{
* Autor para correspondencia PAEZ_2104@hotmail.com
} 
the methodology, then, the results were compared both individually and collectively gathered in the test and post-test; and based on this, it was determined on what aspects this methodology strengthen problem solving competence in high school students. The results were to conclude that the problem-solving competence in the area of mathematics could be strengthened, in the ninth grade, being reflected in the improvement of students' abilities to solve problems in the area and in fundamental geometric figures. The above evidenced in the results of the post test, this serves as taken as a base so that the students can find new knowledge more interesting, promoting the investigation in real contexts, making the meaningful learning easier and generating changes in the reasoning level.

\section{Palavras-chave}

Competência Matemática, Metodologia, Aprendizagem Baseada em Problemas, Resolução de Problemas.

\begin{abstract}
Resumo: No ensino de matemática é indispensável implementar estratégias que permitam aos alunos relacionarse com o seu contexto imediato, visando poder desenvolver significativamente a sua aprendizagem. A presente pesquisa desenvolvese com base na metodologia Aprendizado Baseado em Problemas (ABP), como objetivo de analisar de que maneira influencia o fortalecimento da competência matemática a resolução de problemas. Levando emconsideraçãoum enfoque quantitativo e um projetopré-experimental, realizouse uma análise descritiva da capacidade dos alunos para resolver problemas prévios à metodologia, em seguida, compararamse os resultados tanto individuais como grupais obtidos no teste e no pós-teste; e com base nestes, determinouse os aspectos cuja metodologia fortalece a competência da resolução de problemas nos alunos de em sino médio. Os resultados permitir ame concluise que a competência na resolução de problemas na área de matemática poderia ser reforçada, no nono ano, refletindo na melhoria da capacidade dos alunos para resolver problemas na área e em figuras geométricas fundamentais. $\mathrm{O}$ acima evidenciado nos resultados do pósteste, isto serve como bases para que os alunos dessem sentido a novos conhecimentos, promovendo a reflexão em contextos reais, facilitando a aprendizagem significativa e gerando mudanças no nível de raciocínio.
\end{abstract}

\section{Introducción}

Considerando que no se puede dejar a un lado el papel que desempeña el maestro de matemáticas en el aula y la influencia de su quehacer metodológico en el desarrollo de competencias, es muy interesante revisar la forma como el docente percibe la competencia resolución de problemas en sus encuentros pedagógicos, debido a que podría hallar una descontextualización de las actividades propuestas con el aprendizaje de la matemática. La innovación en la educación debe ser el reto más importante para los docentes y las instituciones, ya que las prácticas tradicionales han fortalecido la creencia de que resolver un problema es se enfatiza en el esquema de solución de problemas: Datos-Operaciones-Resultado.

Efectivamente, diferentes investigaciones exponen que los docentes con frecuencia tienen dificultades para enseñar a los estudiantes cómo abordar los problemas y cómo hacer uso de herramientas adecuadas (Harskamp \& Suhre, 2006), es por ello, que se plantea como posible propuesta de solución a la problemática, la implementación de la metodología ABP como una forma de retar y motivar a los estudiantes a comprometerse en el desarrollo de esta competencia, la búsqueda del conocimiento y de respuestas, no solo a las preguntas que plantean los libros o maestros sino en especial a sus propias preguntas.
Barrows (1986) define al ABP como "un método de aprendizaje basado en el principio de usar problemas como punto de partida para la adquisición e integración de los nuevos conocimientos". De esta manera el ABP se convierte en una experiencia pedagógica de tipo práctico organizada para investigar y resolver problemas vinculados al mundo real, que fomenta 
el aprendizaje activo y lo integra con la vida real, desde una mirada multidisciplinar. Diaz Barriga, (2006).

Por su parte, en educación matemática el término resolución de problemas ha venido tomando importancia a través de los años, debido a que es considerado por los maestros como el corazón de la actividad matemática, reconociéndole su gran influencia en el proceso de enseñanza y aprendizaje en el que viven a diario tanto maestros como estudiantes. Polya $(1945,1968)$ afirma que en la resolución de problemas matemáticos "la principal novedad es apuntar que una estrategia adecuada para resolver problemas considerados muy difíciles, consiste en su fraccionamiento en subproblemas más simples que sí admiten una solución" (Perales, 1993, p.172), y plantea, a su vez, un método heurístico de cuatro pasos a seguir para la resolución de problemas.

Teniendo en cuenta los resultados obtenidos en las evaluaciones de competencias básicas consideradas esenciales para la vida y el buen desempeño en la sociedad, desde la década de los 90, los gobiernos se han propuesto mejorar y redefinir los sistemas educativos en cada país, siendo este un fin de la Organización para la Cooperación y el Desarrollo Económico (OCDE), (Martínez, 2015) y el cual viene lográndose a través de proyectos como el Programa para la Evaluación Internacional de Estudiantes (PISA).

Los resultados en las pruebas realizadas en los últimos años en los países que pertenecena la OCDE, muestran que cerca del $15 \%$ de los estudiantes rinde por debajo del nivel 2, considerado el umbral básico de competencias científicas, y tan solo el 9\% aproximadamente obtiene un rendimiento sobresaliente en la resolución de problemas, dentro de la evaluación de matemáticas de PISA (OCDE, 2016, p. 4). Colombia quedó ubicada en el puesto 57, con un puntaje de 390 puntos en matemáticas y 429 en resolución de problemas (OCDE, 2016, p. 5).
De acuerdo con los puntajes obtenidos por Colombia en las pruebas PISA, en matemáticas se evidencia que más del $70 \%$ de los estudiantes se encuentran por debajo del nivel dos de desempeño, contrastando con la media de $0,275 \%$ de estudiantes en los niveles cinco y seis de desempeño considerado como los más altos, es decir, que la mayoría de los estudiantes no evidencian las competencias mínimas necesarias para desempeñarse en las sociedades contemporáneas.

Por su parte, en los resultados a nivel nacional, según los reportes generados por el ICFES, durante el periodo de 2009 a 2016 en matemáticas el puntaje promedio en la prueba Saber $11^{\circ}$ se encuentra por debajo de los 50 puntos, lo cual refleja que también existen bajos desempeños en el área.

Por otra parte, el caso de los puntajes obtenidos en matemáticas en las pruebas Saber $9^{\circ}$ durante los 3 últimos años (2014 a 2016), mostró que las estrategias que se han incorporado no están dando los resultados esperados, debido que, por ejemplo, el nivel de desempeño "Avanzado" en lugar de aumentar, disminuyó de un 5\% a un 4\%. De igual forma se presentó una disminución en el nivel satisfactorio de $21 \%$ a $20 \%$, el nivel mínimo se mantuvo con el 53\% y el aumento se manifestó en el nivel "Insuficiente" de un 21\% a 23\% (ICFES, 2016).

Estas problemáticas anteriormente expuestas, también se ven reflejadas a nivel local en la ciudad de Cúcuta, donde es evidente, que los bajos resultados en la competencia resolución de problemas es una realidad contraproducente y general que, por ende, preocupa a los maestros de matemáticas. De allí surge la necesidad de iniciar un proyecto de investigación que permita fortalecer estas falencias aplicando la metodología de Aprendizaje Basado en Problemas. El trabajo de campo de desarrollo en estudiantes de noveno grado, buscando dar respuesta al interrogante planteado: ¿Cómo influye la metodología Aprendizaje Basado en Problemas en el fortalecimiento de la competencia de 
resolución de problemas por parte de estudiantes de noveno grado?

\section{Materiales y métodos}

La investigación se fundamentó bajo un modelo de tipo cuantitativo con enfoque descriptivo, ofreciendo la posibilidad de generalizar y comparar resultados. Se hizo un trabajo de tipo pre-experimental teniendo como instrumento recolector de información el cuestionario desarrollado en dos momentos, el test y el post test, es decir el antes y el después de la aplicación de la metodología aprendizaje Basado en Problemas (ABP).

\section{Población y muestra}

El estudio se realizó en estudiantes del grado noveno de una institución educativa de carácter privado de la ciudad de Cúcuta, teniendo una población de 68 estudiantes de grado noveno, de los cuales se tomó una muestra de 25 estudiantes del grado 9-02, mediante un muestro no probabilístico por conveniencia, dicha muestra se tomó debido a las facilidades de acuerdo al horario del docente para realizar los encuentros con el grupo de estudiantes. La investigación se llevó a cabo en tres etapas: la primera con el fin de caracterizar el estado actual de la competencia para resolver problemas, la segunda con el propósito de fortalecer la competencia para resolver problemas con la implementación de la metodología Aprendizaje Basado en Problemas. (ABP), la tercera fase consistió en contrastar la competencia de los estudiantes para resolver problemas antes y después de aplicar la metodología.

Lo anterior se fundamenta en un diseño preexperimental, que para Hernández, Fernández y Baptista (2010), es un tipo de estudio que se caracteriza por trabajar con un solo grupo al cual se le aplica una prueba previa al estímulo o tratamiento experimental, después se le administra el tratamiento y finalmente se le aplica una prueba posterior al estímulo.

\section{Instrumentos}

Los instrumentos utilizados fueron: un cuestionario sobre resolución de problemas y una unidad didáctica correspondiente a la metodología ABP.

El primero con el propósito de evaluar la competencia para resolver problemas en los estudiantes de Noveno grado y desarrollado bajo los criterios del método heurístico de los cuatro pasos de George Polya: comprender el enunciado de un problema, seleccionar estrategias adecuadas en la solución del mismo, ejecutar con precisión los pasos necesarios de un plan de solución, y examinar la solución obtenida. Constaba de 15 preguntas clasificadas en cuatro problemas, 8 preguntas eran de selección múltiple con única respuestas y siete preguntas eran abiertas, los temas utilizados fueron área, volumen y operaciones básicas.

El segundo instrumento fue una unidad didáctica basada en los siete pasos del Aprendizaje Basado en Problemas propuestos por Moust, Bouhuijs y Schmidt (2007) y el modelo aplicado por Fe y Alegría, creado por Benjumea (2013). Se inició con una situación problema derivada de una necesidad del contexto. Un problema abierto y débilmente estructurado, integrando varias asignaturas, de esta manera se buscaba dar solución al problema planteado.

\section{Procedimiento y análisis}

Para la sistematización de los datos, se realizó un análisis descriptivo de los resultados obtenidos en el test y el post test, junto con comparaciones estadísticas mediante métodos no paramétricos entre los resultados de las dos pruebas, mediante la utilización del software SPSS. El análisis descriptivo de los resultados obtenidos por los estudiantes implicó la elaboración de tablas y gráficas como diagramas de barras, las barras de error y tablas con estadísticas descriptivas, posteriormente se realizó prueba de Kolmogorov Smirnov para establecer la normalidad de las variables; dado que se deduce que tienen comportamiento normal al aceptarse la hipótesis 
nula; lo cual permite la aplicación de pruebas de muestras relacionadas donde se demostró que la relación entre las medias es estadísticamente significativa, es decir la metodología Aprendizaje Basado en Problemas tuvo efecto significativo sobre la competencia resolución de problemas influyendo positivamente en el mejoramiento de la misma. Finalmente se realizó análisis comparativo de los resultados obtenidos antes y después de la aplicación de la metodología ABP.

\section{Resultados y discusión}

Para la clasificación de los resultados obtenidos en el cuestionario, se desarrolló una escala de valoración diseñada por el autor con base en una plantilla de evaluación de la competencia resolución de problemas creada por la universidad Politécnica de Madrid y la escala de valoración utilizada en Colombia por el ICFES en las pruebas saber noveno.

En primera instancia se analizó el nivel de competencia que poseían los estudiantes de noveno grado en resolución de problemas en matemáticas, la tabla 1, tabla 2, tabla 3 y tabla 4 muestran el análisis realizado con base en los resultados obtenidos en el test, teniendo en cuenta las cuatro fases del método heurístico de Polya, mencionadas anteriormente.

Tabla 1. Fase comprensión de un problema

\begin{tabular}{|c|c|}
\hline RESULTADOS & DISCUSIÓN \\
\hline $\begin{array}{l}\text { Identifican bien la información dada, los datos, la condición y los símbolos, pero } \\
\text { se les dificulta usarlos correctamente. } \\
\text { Representan información de manera gráfica, de forma incompleta o desorganizada. }\end{array}$ & $\begin{array}{c}\text { La primera fase consiste en identificar qué es lo que hay que hacer, la } \\
\text { información dada, los datos, los contenidos que se necesitarán, la con- } \\
\text { dición, los símbolos y las palabras en que están planteadas, identificar } \\
\text { las dificultades y cambiar el formato de presentación del problema } \\
\text { usando gráficos. (Polya, 1945) }\end{array}$ \\
\hline $\begin{array}{l}\begin{array}{l}\text { Dificultad para señalar si una condición es suficiente para dar solución a un pro- } \\
\text { blema. }\end{array} \\
\text { Habilidad para detectar datos innecesarios en los problemas. }\end{array}$ & $\begin{array}{l}\text { Los bajos niveles de comprensión de los enunciados es una de las } \\
\text { mayores falencias en el proceso de resolución de problemas. (Boscan } \\
\text { y Klever, 2012) }\end{array}$ \\
\hline
\end{tabular}

Fuente: Autor, Proceso de investigación

Tabla 2. Fase concepción de un plan

\begin{tabular}{|c|c|}
\hline RESULTADOS & DISCUSIÓN \\
\hline $\begin{array}{c}\text { Buen desempeño al momento de verificar que los resultados obtenidos dieran } \\
\text { respuesta a la pregunta realizada. }\end{array}$ & $\begin{array}{l}\text { Según Polya, esta fase consiste en verificar el resultado, verificar el razona- } \\
\text { miento y preguntarse si se puede emplear este mismo método en algún otro } \\
\text { problema. }\end{array}$ \\
\hline $\begin{array}{l}\text { Poca habilidad para identificar casos similares en los que podría usarse un } \\
\text { razonamiento similar. }\end{array}$ & $\begin{array}{l}\text { Boscan y Klever (2012), afirman en su investigación "los estudiantes tienen } \\
\text { la idea de que todos los problemas se solucionan a través de una adición, }\end{array}$ \\
\hline $\begin{array}{l}\text { Dificultad al identificar la respuesta que corresponde a un problema determi- } \\
\text { nado. }\end{array}$ & $\begin{array}{c}\text { efectúan operaciones sin reflexionar si estas conllevan a obtener la respuesta } \\
\text { correcta". }\end{array}$ \\
\hline $\begin{array}{l}\text { Habilidades escogiendo un enunciado correcto de acuerdo a una respuesta } \\
\text { dada. }\end{array}$ & \\
\hline
\end{tabular}

Fuente: Autor, Proceso de investigación

Tabla 3. Fase ejecución de un plan

\begin{tabular}{|c|c|}
\hline RESULTADOS & DISCUSIÓN \\
\hline $\begin{array}{l}\text { Buen desempeño al momento de ordenar los pasos a seguir en una estrategia } \\
\text { de solución. }\end{array}$ & $\begin{array}{l}\text { Concebir un plan es saber que razonamientos, operaciones o construcciones } \\
\text { se deben desarrollar para dar solución al problema, realizar aproximaciones } \\
\text { y valorar las posibles estrategias de abordaje del problema.(Polya, 1945) }\end{array}$ \\
\hline $\begin{array}{l}\text { Altos niveles en aproximaciones de resultados en operaciones. } \\
\text { Dificultad para seleccionar estrategias correctas y pertinentes que den } \\
\text { solución a un problema. }\end{array}$ & $\begin{array}{l}\text { Ante la ausencia de un plan, los estudiantes tienden a echar mano de estrate- } \\
\text { gias irreflexivas que en la mayoría de los casos desembocan en errores, por } \\
\text { esta razón es un paso fundamental que permite alcanzar un objetivo definido } \\
\text { (Silva y Rodríguez,2011) }\end{array}$ \\
\hline $\begin{array}{c}\text { Dificultad para crear planes de solución de acuerdo a los datos y preguntas } \\
\text { del problema. }\end{array}$ & \\
\hline
\end{tabular}

Fuente: Autor, Proceso de investigación 
Tabla 4. Examinar la solución obtenida

\begin{tabular}{|c|c|}
\hline RESULTADOS & DISCUSIÓN \\
\hline $\begin{array}{l}\text { La mayor habilidad fue para identificar datos y reemplazarlos correctamente } \\
\text { en fórmulas. } \\
\text { Plantean de manera parcial o errónea enunciados con base en operaciones. }\end{array}$ & $\begin{array}{l}\text { Ejecutar el plan propone que al realizarlo debe comprobarse cada uno de los } \\
\text { pasos, asegurarse de que sean correctos, seguir el plan teniendo cuidado en } \\
\text { el reemplazo de fórmulas o en el procedimiento de las operaciones. (Polya, } \\
\text { 1945) }\end{array}$ \\
\hline $\begin{array}{c}\text { Facilidad para reconocer errores en procedimiento u operaciones planteadas. } \\
\text { Ejecutan correctamente en su mayoría los procedimientos de cálculo, en } \\
\text { operaciones básicas. }\end{array}$ & $\begin{array}{l}\text { Arteaga y Guzmán (2005) resaltan la importancia de trabajar con problemas } \\
\text { de diferente naturaleza para estimular el desarrollo de estrategias y habilida } \\
\text { des, y de esta manera se desarrolle la capacidad de responder a la ejecución } \\
\text { de diferentes procedimientos. }\end{array}$ \\
\hline
\end{tabular}

Fuente: Autor, Proceso de investigación

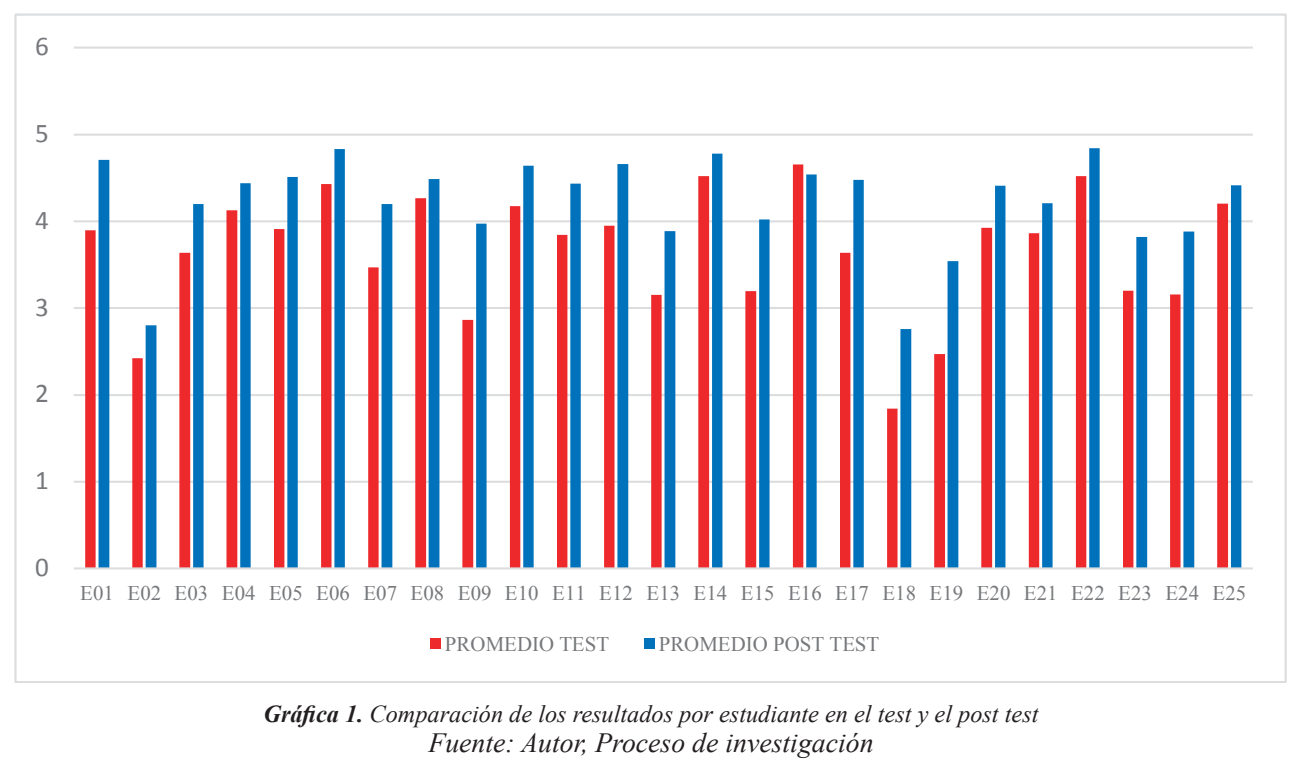

De lo anterior se deduce que la competencia resolución de problemas en el área de matemáticas, se pudo fortalecer en el grado noveno, permitiendo a los estudiantes ser más organizados al momento de resolver un problema, diseñando un plan, ejecutando las actividades planeadas y evaluando la validez de las soluciones obtenidas. La gráfica 2 muestra una comparación general de los resultados

en el test y el post test para cada una de las fases propuestas por Polya. De ella se destaca que el promedio grupal mejoró significativamente en cada una de las cuatro fases evaluadas, potenciando además de las competencias y habilidades individuales en cada estudiante, destrezas como el trabajo en equipo, asignación de roles, seguimiento de instrucciones y liderazgo.

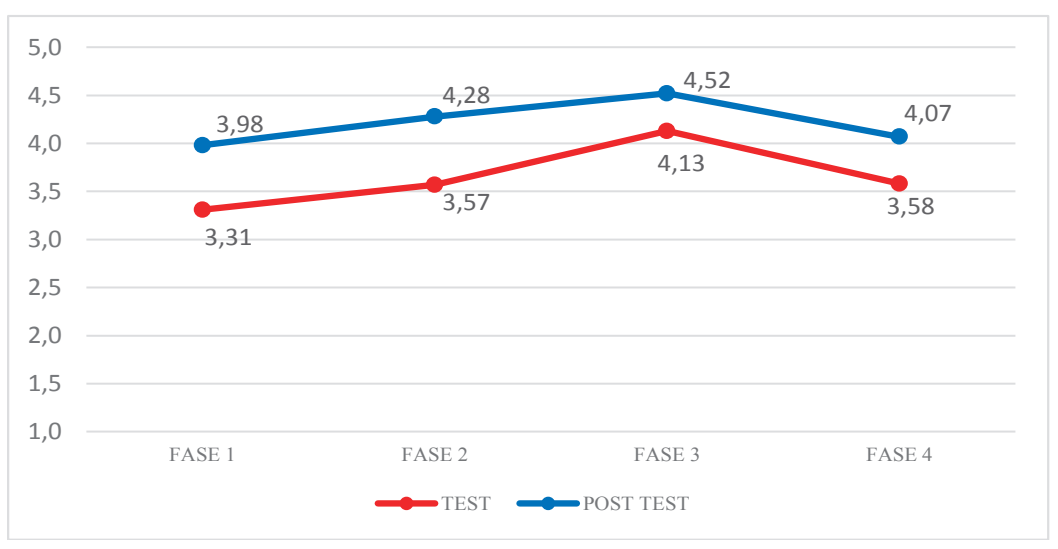

Gráfica 2. Comparación de los resultados por fase en el test y el post test Fuente: Autor, Proceso de investigación 
Respecto al mejoramiento del desempeño en cada fase, luego de la implementación de la metodología $\mathrm{ABP}$, algunos autores como Boscán y Klever (2012) llegaron a la misma conclusión, al aplicar dicha metodología, lograron que el número de estudiantes que comprendían los enunciados de los problemas aumentará, por consiguiente, también aumentará el número de respuestas correctas. Así mismo, comprobaron que posterior a la intervención, la capacidad de reflexión, razonamiento y argumentación se fortaleció en los estudiantes.

$\mathrm{Al}$ analizar el avance que tuvieron las cuatro fases, puede afirmarse que la lectura comprensiva de la situación problema realizada en cada equipo y de manera general fue fundamental. De igual manera las actividades desarrolladas a través de la unidad didáctica, tales como tener que proponer ideas, argumentar y socializar con los demás miembros del equipo para llegar a una decisión común, permitieron potenciar las habilidades de los estudiantes. En los hallazgos de Hidalgo, Mera y López (2015), los estudiantes mostraron mayor habilidad al solucionar problemas luego de la intervención, notándose mejoría en el interés por investigar, y mayor comprensión de los saberes aprendidos, caso muy similar a lo sucedido con esta investigación.

En tercer lugar, se determinó de qué manera la metodología ABP fortaleció la competencia en resolución de problemas del grupo de estudiantes. Los hallazgos evidencian que, si existió una influencia concluyente en cada una de las cuatro fases, evidenciando avances significativos en las cuatro fases de Polya, específicamente al momento del post test, demostrando que los estudiantes luego de la metodología mejoraron su desempeño. Por lo tanto, se puede afirmar que existió una influencia positiva entre el proceso aplicado en el ABP y la habilidad de resolución de problemas en los estudiantes de noveno grado de la institución educativa.En esta investigación se planteó como hipótesis que la implementación de la metodología Aprendizaje Basado en Problemas si influye en el desarrollo de la competencia para resolver problemas en los estudiantes de grado noveno. Por tal razón se aplicó la Prueba t para dos muestras relacionadas, con el fin de comprobar si los puntajes obtenidos inicialmente con el test en cada una de las fases de la resolución de problemas, difieren significativamente de las puntuaciones conseguidas en las fases correspondientes durante el post test luego de finalizar la metodología ABP. La tabla 5 muestra que sí hay diferencias significativas en todas las fases, puesto que la significación bilateral es menor que 0.05 , demostrando que la relación es estadísticamente significativa, es decir la metodología $\mathrm{ABP}$ tuvo efecto significativo sobre la competencia resolución de problemas.

\begin{tabular}{ccc}
\multicolumn{3}{c}{ Tabla 5. Prueba de muestras relacionadas } \\
\hline FASE & MEDIA & SIGNIFICACIÓN BILATERAL \\
\hline FASE 1 TEST & $-0,6720$ & 0,000 \\
FASE 1 POST TEST & $-0,7066$ & 0,014 \\
FASE 2 TEST & & \\
FASE 2 POST TEST & $-0,3900$ & 0,000 \\
FASE 3 TEST & & 0,010 \\
FASE 3 POST TEST & $-0,4933$ & \\
FASE 4 TEST & & \\
FASE 4 POST TEST & & \\
\hline
\end{tabular}

Fuente: Autor, Proceso de investigación

\section{Conclusiones}

Aprendizaje Basado en Problemas es una metodología que cree y apoya los principios de la educación actual, en la cual lo más importante es que los estudiantes se apropien de competencias básicas que le permitan navegar con éxito en los mares del conocimiento, ser aptos ante cualquier circunstancia para resolver problemas, por lo tanto permite que el proceso se centre en el estudiante, siendo el mismo quien desarrolla competencias propias que le permiten enfrentarse con mayor facilidad ante cualquier situación problema no solo comprendiéndolo, sino proponiendo posibles soluciones, ideando planes para llegar a ella, examinando de manera minuciosa el trabajo 
realizado, lo cual le permite un rango mínimo de equivocación.

Debe resaltarse que, esta metodología surgió como idea de educadores con el propósito de incrementar habilidades para la solución de problemas de estudiantes universitarios, es decir, habilidades para adquirir información, organizarlas en diversas hipótesis y probarlas por medio de nueva información. Así que, con base en los resultados obtenidos puede afirmarse que esta metodología cumple con los fines que le fueron propuestos.

Tal como lo plantea el MEN (1998), las situaciones problemáticas son el contexto más oportuno para desarrollar procesos de pensamiento, y conferir sentido al aprendizaje de las matemáticas, al relacionarla con la vida diaria, lo que hace que la resolución de problemas no debe ser el final del proceso de enseñanza, sino en realidad, su partida. Los resultados también permiten corroborar que en el ABP el tutor hace que el nivel de desarrollo del estudiante sea mayor solo con su orientación, hasta lograr mejorar autonomía en él, es decir, el tutor guía a los estudiantes a una reflexión que les permita reconocer lo que necesitan aprender por medio del descubrimiento, tomar sus propias decisiones, a relacionar los nuevo con experiencias anteriores y con sus propios intereses, en otras palabras, es una metodología que va de la mano con las teorías de la zona del desarrollo próximo de Vygotsky, la teoría de la autodeterminación de Edwar Deci y Richard Ryan, y el aprendizaje significativo de Ausubel.

Aspectos como los bajos niveles de comprensión lectora de los estudiantes, la apatía o el desconocimiento de las acciones a realizar, el hábito de los docentes a no trabajar de manera interdisciplinar, y el desconocimiento de la metodología ABP fueron algunas circunstancias limitantes para esta investigación, pero que de alguna manera se pudo aportar también al fortalecimiento de cada una de ellas. Por consiguiente se puede extender esta investigación al desarrollo de las demás competencias del área de matemáticas, como también en las demás áreas, el desarrollo de competencias ciudadanas y/o laborales o las variables relacionadas con la capacidad de resolución de problemas en los diferentes niveles educativos.

\section{Referencias}

Barrows H.S. (1986) A Taxonomy of problem based learning methods, Medical Education, 20, 481-486.

Boscán, M., Klever, K. (2012). Metodología basada en el método heurístico de Polya para el aprendizaje de la resolución de problemas matemáticos. Revista Escenarios, 10 (2), 7-19.

Díaz Barriga, F, (2006). Aprendizaje Basado en Problemas. De la teoría a la práctica. Revista electrónica: Perfiles Educativos, 28, 111.

George Polya (1945). Cómo plantear y resolver problemas [título original: How To Solve It?]. México: Trillas. 215 pp.

Harskamp, E. and Suhre, C. (2006). Improving mathematical problem solving: A computerized approach. En: Computers in Human Behavior, 22.

Hernández, R., Fernández, C., \& Baptista, P. (2010). Metodología de la investigación. México: Mc Graw Hill.

Hidalgo, H., Mera, E., López, J. (2015).Aprendizaje basado en problemas, como potencializador del pensamiento matemático. (Tesis de Maestría en Educación desde la Diversidad, Universidad de Manizales, Manizales). Recuperado de http://190.242.114.6/vufind/Record/RUM b15910fe7297ab76646a17a89eb9a22c

Icfes interactivo. 2016. Página web oficial http://www2.icfesinteractivo.gov.co/ ReportesSaber359/seleccionListaInstituciones. jspx

Moust, J; Bouhuijs, P; Schmidt, H. (2007). El aprendizaje basado en problemas: Guía del estudiante. Cuenca: Ediciones de la UCLM 
Ministerio de Educación Nacional

(MEN). (2003). Estándares básicos de competencias en matemáticas. Recuperado de http://eduteka.icesi.edu.co/pdfdir/ MENEstandaresMatematicas2003.pdf

Ministerio de Educación Nacional (MEN). (2010). Pruebas Saber $3^{\circ}, 5^{\circ}$ y $9^{\circ} .04$ de octubre de 2016, Sitio web: http://www.mineducacion. gov.co/1759/w3-article-244735.html

Ministerio de Educación Nacional (MEN). (2014). Lineamientos Curriculares Matemáticas. Magisterio, Bogotá. Tomado de http://www.mineducacion.gov.co/1621/ articles-89869_archivo_pdf9.pdf

Organización para la Cooperación y el Desarrollo Económicos (OCDE). PISA 2105 resultados clave. OCDE. 2016. https://www.oecd.org/ pisa/pisa-2015-results-in-focus-ESP.pdf

Perales, F. (1993). La resolución de problemas. Una Revisión Estructurada, 11(2), 170-178. Recuperado de: https://ddd.uab.cat/pub/edlc/02 124521v11n2/02124521v11n2p170.pdf

Polya, G. Estrategias para la solución de problemas. Recuperado de http://www. winmates. net/includes/polya. php (octubre 2009).

POLYA, G. (1980). On Solving Mathematical Problem in High Scholl. En KRULIK, S. y REIS, R. (Eds.) Problem Solving in School Mathematics. NCTM: Reston. [92] 